Revue d'histoire de l'Amérique française

REVUE D.HISTOIRE DE L'AMÉRIQUE FRANÇAISE

\title{
La traite des fourrures en Haute-Mauricie avant 1831. Concurrence, stratégies commerciales et petits profits
}

\section{Claude Gélinas}

Volume 51, numéro 3, hiver 1998

URI : https://id.erudit.org/iderudit/005441ar

DOI : https://doi.org/10.7202/005441ar

Aller au sommaire du numéro

\section{Éditeur(s)}

Institut d'histoire de l'Amérique française

\section{ISSN}

0035-2357 (imprimé)

1492-1383 (numérique)

Découvrir la revue

\section{Citer cet article}

Gélinas, C. (1998). La traite des fourrures en Haute-Mauricie avant 1831.

Concurrence, stratégies commerciales et petits profits. Revue d'histoire de l'Amérique française, 51(3), 391-417. https://doi.org/10.7202/005441ar
Résumé de l'article

Cet article présente un historique du commerce des fourrures en Haute-Mauricie depuis l'implantation des premiers postes de traite jusqu'en 1831. Il est d'abord fait état des motivations qui ont amené des petits commerçants indépendants, puis la North West Company, à s'établir dans cette région. Par la suite, l'attention est portée sur la concurrence qui a sévi en Haute-Mauricie, durant les années 1820, entre la Hudson's Bay Company et la King's Posts Company. Il est proposé que les activités des deux compagnies dans la région étaient davantage motivées par des considérations d'ordre stratégique, à savoir la protection des frontières de leurs monopoles respectifs, que par la recherche de profits. 


\title{
LA TRAITE DES FOURRURES EN HAUTE-MAURICIE AVANT 1831. CONCURRENCE, STRATÉGIES COMMERCIALES ET PETITS PROFITS
}

\author{
CLAUDE GÉLINAS \\ Département d'anthropologie \\ Université de Montréal
}

\begin{abstract}
RÉSUMÉ
Cet article présente un historique du commerce des fourrures en Haute-Mauricie depuis l'implantation des premiers postes de traite jusqu'en 1831. Il est d'abord fait état des motivations qui ont amené des petits commerçants indépendants, puis la North West Company, à s'établir dans cette région. Par la suite, l'attention est portée sur la concurrence qui a sévi en Haute-Mauricie, durant les années 1820, entre la Hudson's Bay Company et la King's Posts Company. Il est proposé que les activités des deux compagnies dans la région étaient davantage motivées par des considérations d'ordre stratégique, à savoir la protection des frontières de leurs monopoles respectifs, que par la recherche de profits.
\end{abstract}

\section{ABSTRACT}

This paper presents a history of the fur trade in the Upper St.Maurice region from the time of the first trading posts up to 1831. First, the motivations of the early traders and those of the North West Company for entering this region are discussed. Greater attention will be given to the opposition between the Hudson's Bay Company and the King's Posts Company during the 1820's. It is suggested that the activities of both companies in the Upper St.Maurice region were more oriented towards the protection of each one's monopoly them towards making profits.

Pour des raisons qu'il faudra un jour cerner, la Haute-Mauricie n'a jamais réellement suscité l'intérêt des chercheurs et ce, dans pratiquement tous les domaines. On ne connaît que très partiellement sa géomorphologie. L'essentiel de sa faune reste à être inventoriée. Les études ethnographiques d'envergure sont rares et datent, pour plusieurs, du début du siècle. Enfin, l'histoire de la région reste à écrire, bien que des défrichages aient déjà été entrepris, surtout en ce qui a trait au passé des 
groupes autochtones ${ }^{1}$. Or, particulièrement sur le plan historique, non seulement les données relatives à la Haute-Mauricie sont plus abondantes qu'il n'y paraît à première vue, mais elles révèlent une histoire régionale peu banale. C'est particulièrement le cas en ce qui concerne la période qui s'étend de 1774 à 1831, durant laquelle la région était encore essentiellement branchée sur l'économie des fourrures. Pourtant située au cœur du territoire québécois, la Haute-Mauricie fut une des dernières régions à accueillir des postes de traite et les différents commerçants qui s'y sont implantés, au fil des ans, se sont livré des luttes souvent féroces, et pas toujours dans le seul but d'accumuler des ballots de fourrures.

Le présent article a pour but de retracer les motivations qui ont amené divers commerçants de fourrures à s'établir en Haute-Mauricie, de même que leurs manières de mener leurs activités économiques avant 1831. Il sera particulièrement question du contexte qui prévalait au cours des années 1820, alors que deux grands monopoles ont coexisté dans la région, à savoir la Hudson's Bay Company et la King's Posts Company ${ }^{2}$. Les livres de comptabilité des postes que les deux compagnies ont opérés à Weymontachie sont pratiquement tout ce qui

1. Norman Clermont, Ma femme, ma hache et mon couteau croche. Deux siècles d'histoire à Weymontachie (Québec, Ministère des Affaires culturelles, 1977); Jean Baribeau, Les missions sauvages du Haut Saint-Maurice au XIXe siècle, mémoire de maîtrise (théologie), Université du Québec à Trois-Rivières, 1978); Raynald Parent, Histoire des Amérindiens, du Saint-Maurice jusqu'au Labrador: de la préhistoire à 1760, thèse de doctorat (histoire), Université Laval, 1985); Bernard Allaire, Une économie en déséquilibre: les autochtones du Saint-Maurice, de la traite des fourrures à la construction des barrages hydroélectriques, mémoire de maîtrise (histoire), Université Laval, 1987); Maurice Ratelle, Contexte historique de la localisation des Attikameks et des Montagnais de 1760 à nos jours (Québec, Ministère de l'Énergie et des Ressources, 1987); Claude Gélinas, «Identité et histoire des autochtones de la Haute-Mauricie aux XVIIe et XVIIIe siècles: un regard sur le débat Attikamègues - Têtes de Boule», dans R. Tremblay, dir., L'éveilleur et l'ambassadeur. Essais archéologiques et ethnohistoriques en hommage à Charles A. Martijn (Montréal, Recherches amérindiennes au Québec), à paraître.

2. Au temps du Régime français, le Domaine du roi, un vaste territoire qui s'étendait du bassin du Saguenay-Lac-Saint-Jean jusqu'à la Côte-Nord, était réservé à la traite des fourrures. Il était loué à des exploitants qui remettaient une part importante de leurs revenus au roi de France. À la suite de la Conquête, les autorités anglaises ont maintenu le système d'affermage de ce qui prenait désormais le nom de King's Posts. Â ce sujet, voir Nelson-Martin Dawson, Lendemains de Conquête au royaume du Saguenay. Le Domaine du roi dans la politique impériale britannique (1760-1767) (Montréal, Nuit blanche, 1996). En 1802, ce fut la North West Company qui loua les King's Posts, jusqu'à sa fusion avec la Hudson's Bay Company en 1821. L'année suivante, un groupe d'associés, avec à leur tête John Goudie - remplacé par William Lampson à compter de 1828 -, obtinrent le bail du Domaine en offrant plus d'argent pour la location que la Hudson's Bay Company. Voir John S. Galbraith, The Hudson's Bay Company as an Imperial Factor, 1821-1869 (Toronto, University of Toronto Press, 1957), 27-29. Les exploitants du Domaine furent fréquemment désignés dans la littérature de l'époque sous le nom de King's Posts Company. 
reste pour témoigner des modalités entourant la concurrence commerciale en Haute-Mauricie à cette époque ${ }^{3}$. Néanmoins, ils fournissent suffisamment d'informations sur les politiques de prix en vigueur, sur l'importance du système de crédit et sur les comportements économiques des autochtones pour démontrer que, si la Hudson's Bay Company semble avoir nettement triomphé sur le plan de l'acquisition des fourrures, les deux compagnies se sont livré un combat beaucoup plus serré sur le plan de ce qui, en premier lieu, justifiait la présence des établissements de traite en Haute-Mauricie, à savoir la protection des frontières de la Terre de Rupert et du Domaine du roi.

L'ARRIVÉE DES COMMERÇANTS EN HAUTE-MAURICIE, 1774-1821

À compter de la fondation de Trois-Rivières en 1634, un certain nombre d'autochtones de la Haute-Mauricie avaient pris l'habitude de descendre la rivière Saint-Maurice pour venir échanger leurs fourrures avec les marchands locaux. Or, dès le mitan du XVII ${ }^{\mathrm{e}}$ siècle, il était devenu fréquent que des petits commerçants fassent le chemin contraire et s'aventurent à remonter la rivière, afin d'intercepter les chasseurs nomades et leurs fourrures avant qu'ils ne gagnent le poste trifluvien ${ }^{4}$. De même, au lendemain de la Conquête, les généraux anglais Burton et Haldimand, soucieux de protéger la principale activité économique de la ville, d'assurer la libre concurrence pour les marchands trifluviens et d'éviter de se mettre à dos les autochtones qui se plaignaient d'être privés d'un pouvoir de marchandage, interdirent cette pratique, sous peine de punitions sévères ${ }^{5}$. La volonté des autorités de favoriser un commerce fixe avec les autochtones, notamment en limitant le nombre de permis et en astreignant les détenteurs à des paroisses précises, n'empêcha pas, semble-t-il, certains commerçants d'aller solliciter les autochtones sur leur propre territoire. Ainsi, en 1765, Aaron Hart engagea deux hommes: «[...] pour faire le voyage dans la rivière des Trois Riviè-

3. Deux livres de comptabilité appartenant au King's Post de Weymontachie ont été conservés dans les archives de la Hudson's Bay Company. Le premier, qui couvre la période de 1825 à 1828 , se retrouve sous la cote B. 230/d/6, alors que le second, qui couvre la période de 1829 à 1831, est coté $\mathrm{B} .230 / \mathrm{d} / 14$.

4. Reuben G. Thwaites, dir., The Jesuit Relations and Allied Documents. Travels and Explorations of the Jesuit Missionaries in New France, 1610-1791 (Cleveland, The Burrows Brothers Company, 1896-1901), 69: 114; Pierre-George Roy, dir., Inventaire des ordonnances des intendants de la Nouvelle-France conservés aux Archives provinciales de Québec (Beauceville, L'Éclaireur, 1919), 1: 284, 2: 203; Charles-Henri Laverdière et Henri-Raymond Casgrain, dir., Le journal des jésuites (Québec, Brousseau, 1871), 208, 217.

5. Rapport des Archives publiques du Canada (1918): app. B, 257, 258, 271, 283, 285, $313,315,341,343$ 
res en un canot chargé de marchandises pour la traite des Sauvages tetes de boule tant et si loin qu'il conviendra [...] et si besoin en jusque leur séjour ordinaire $[\ldots]^{6}$.» Bien que l'absence de données ne permette pas de documenter adéquatement les actions de ces petits commerçants, il est probable que de telles expéditions dans l'arrière-pays étaient fréquentes à l'époque. Il est aussi possible que les autochtones du Haut Saint-Maurice aient été en contact avec des commerçants installés, notamment, le long des rivières Dumoine et du Lièvre ${ }^{7}$.

On doit à des marchands indépendants, qui plus tard seront rattachés à la North West Company, l'initiative de construire les premiers postes de traite en Haute-Mauricie. Dès 1775, un de ces postes aurait été en opération à Ushkisketa $\left(\right.$ Kikendatch $\left.^{8}\right)$. Cette date est significative puisqu'elle correspond à l'époque où fut ouvert à la colonisation l'ancien territoire réservé aux autochtones en vertu du Traité de Paris, lequel englobait une portion de la Haute-Mauricie. En 1786, trois licences auraient été octroyées à des marchands pour la traite à la rivière SaintMaurice et, en 1802, la North West Company possédait trois postes le long de la rivière, dont un devait être établi à l'embouchure de la Rivière-aux-Rats ${ }^{9}$. En 1806, la même compagnie fit construire un poste à Weymontachie, tout en exploitant un autre poste au lac Negagaming sur la rivière Ruban, près de la source de la rivière Gatineau ${ }^{10}$. Enfin, le marchand François Verreault avait mentionné l'existence d'un poste à Coocoocache au début du $\mathrm{XIX}^{\mathrm{e}}$ siècle $^{11}$. Or, cette affirmation paraît

6. Contrat d'engagement de deux voyageurs par Aaron Hart, 25 avril 1765. Archives du Séminaire de Trois-Rivières, Fonds Aaron-Hart, H-36.

7. Lettre de François Dumoulin à François Baby, Montréal, août 1779. Archives de l'Université de Montréal (AUM), Collection Baby, u/3856; Lettre de Marguerite Dumoulin à François Baby, 1779. AUM, Collection Baby, u/3857; Lettre de Charles Phillips à Sam. Gerrard, Rivière Dumoine, 4 septembre 1795. AUM, Collection Baby, u/9879.

8. Témoignage de Pascal Taché, Journaux de la Chambre d'Assemblée du Bas-Canada (JCABC) (1823-1824), vol. 33: app. R. À l'hiver de 1779-1780, il y avait des postes de traite à Weymontachie et à la Rivière-aux-Rats qui étaient exploités illégalement par des commerçants indépendants. Ceux-ci ont été arrêtés en 1780, et on ignore ce qu'il est advenu des établissements par la suite. Voir Archives nationales du Canada, British Library, Haldimand Papers, Correspondence with M. Tonnancour, Col. of Militia at Trois-Rivières, 1778-1784. MG-21, Add. Mss. 21830 (B170), 121, 142.

9. Louis-P. Cormier, Jean-Baptiste Perreault marchand voyageur parti de Montréal le $28 e$ de mai 1783 (Montréal, Boréal Express, 1978), 104; Gordon C. Davidson, The North West Company (New York, Russell \& Russell, 1967 [1918]), 26, 88, 280.

10. Louis-P. Cormier, op. cit., 105, 107; John Ingall, Rapport des Commissaires nommés en vertu de l'Acte de la 9è Geo. IV, Chap. 29 pour explorer cette partie de la Province qui se trouve entre les rivières Saint-Maurice et Ottawa et qui est encore demeurée déserte et sans culture (Québec, Neilson and Cowan, 1830), 216.

11. Témoignage de François Verreault, JCABC (1823-1824), vol. 33: app. R. 
problématique. Ce poste n'apparaît pas sur la carte de Jean-Baptiste Perreault de 1806, et celui-ci, qui campa à Coocoocache cette même année, n'a pas mentionné dans son récit la présence d'un poste ou de ruines. On pourrait présumer que l'établissement ait été construit peu après le passage de Perreault. Toutefois, en 1829, le lieutenant Ingall, qui passa lui aussi par Coocoocache, n'a dit mot sur l'existence d'un poste à cet endroit. Néanmoins, Verreault est un informateur fiable et, compte tenu que le site de Coocoocache était situé sur une route de portage traditionnelle fort achalandée, l'existence d'un poste de traite à cet endroit ne saurait étonner.

On notera que les premières cartes géographiques de la Haute-Mauricie indiquent la présence de postes de traite dont les documents écrits ne font aucune mention. Sur la carte de Bouchette fils de 1831, on retrouve un «Pisnoy Post» situé à quelques kilomètres en aval du lac Kikendatch. S'y trouve également, à la même latitude que le lac Obiduan [Obedjiwan] et à la longitude $75^{\circ} 50^{\prime}$, un «Old Indian Post». Ces postes ont-ils existé, et, si oui, quand ont-ils été construits et qui les opérait? Bouchette n'a pas remonté la rivière Saint-Maurice en amont de La Tuque, de sorte que son tracé du Haut Saint-Maurice a été réalisé à partir de témoignages d'informateurs. Un de ceux-ci a sans doute été Baptiste Crête, un employé de la King's Posts Company «who appeared intimately acquainted with the St.Maurice, as far as Obiguan [Obedjiwan $]^{12}$ », et que Bouchette avait rencontré à l'été de 1828. Or, nulle part n'est-il fait état de l'existence d'un poste entre Obedjiwan et Weymontachie à la fin des années 1820 . Crête faisait-il référence à un poste plus ancien? Le fait que Verreault ne mentionne pas le Pisnoy Post pourrait indiquer qu'il s'agissait d'un poste antérieur au $\mathrm{XIX}^{\mathrm{e}}$ siècle. Il serait alors curieux que Crête en ait fait état. Une autre explication serait que Bouchette ait voulu indiquer un portage et, au lieu de lire «post», il faudrait lire «port». Toutefois, ailleurs sur sa carte, l'arpenteur a toujours écrit le mot portage sans abréviation. Bref, bien qu'elle soit problématique, l'existence possible d'autres postes que ceux de la North West Company pourrait signifier que le commerce des fourrures en HauteMauricie, au début du XIX ${ }^{\mathrm{e}}$ siècle, ait été plus actif qu'on ne le croit généralement.

Quels objectifs sous-tendaient-ils la présence des commerçants en Haute-Mauricie? Dans les années 1770, le réseau du commerce des fourrures dans la péninsule Québec-Labrador était déjà bien établi. Les

12. Journal of the St.Maurice Exploring Party. JCABC (1829), vol. 38: app. V. 
FIGURE 1

LES POSTES DE TRAITE EN HAUTE-MAURICIE ENTRE 1775 ET 1831

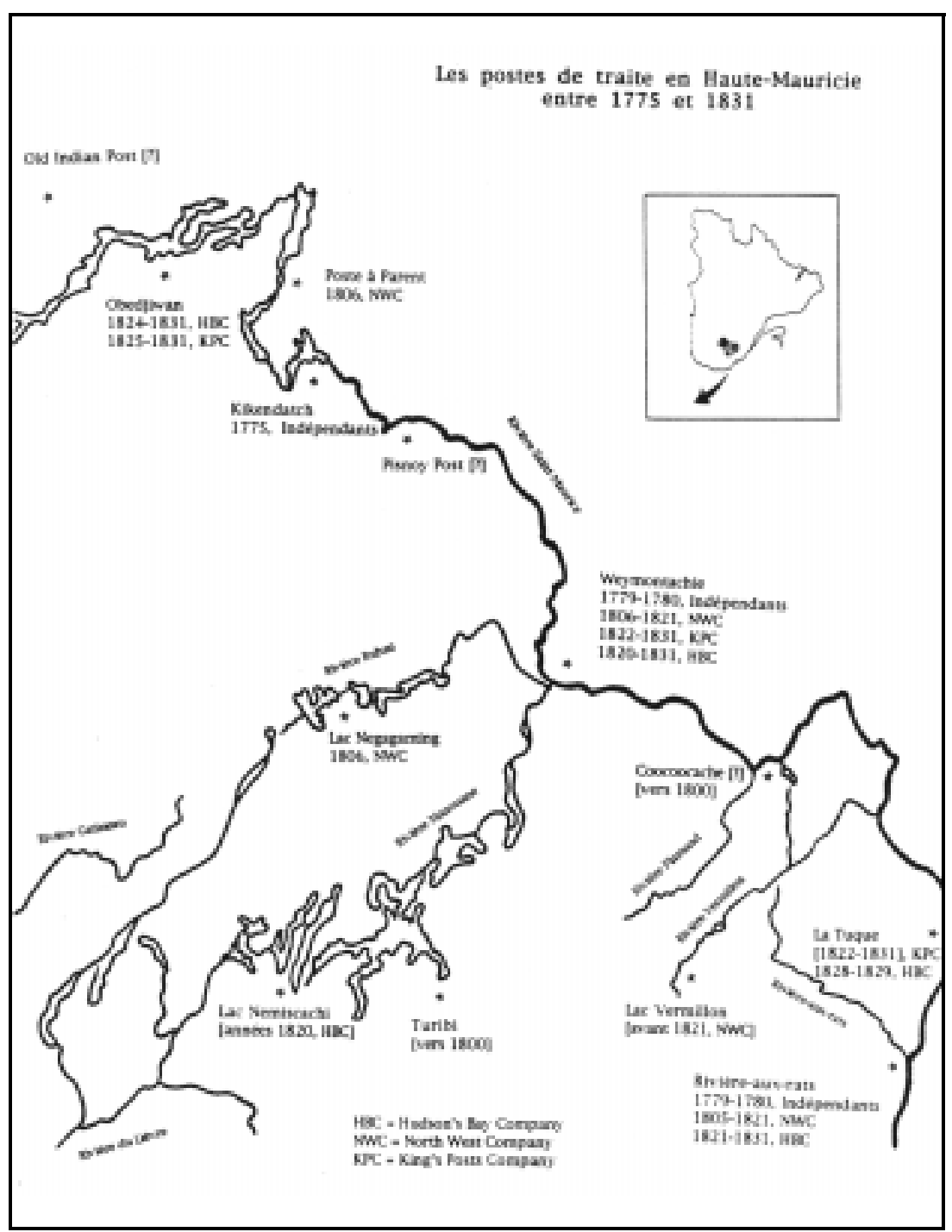

D'après la carte de E. Taché, Carte de la partie nord-ouest de la province de Québec, 1880. 
bassins du Saguenay_Lac-St-Jean, de la baie James et de l'Outaouais, de même que la vallée du Saint-Laurent, étaient depuis longtemps pourvus en postes de traite. En ce sens, la Haute-Mauricie paraissait jusquelà comme une sorte de no man's land, un territoire apparemment sousexploité par les commerçants. Tout de même, cette région était occupée et exploitée par des autochtones qui n'étaient pas étrangers au commerce des fourrures et qui fréquentaient depuis longtemps les établissements de traite situés dans les régions circonvoisines. Aussi, la décision d'établir des postes en Haute-Mauricie n'était pas motivée par la volonté d'y rejoindre une nouvelle clientèle autochtone restée étrangère au commerce. On espérait plutôt inciter les autochtones à ne plus quitter la Haute-Mauricie pour troquer leurs fourrures et, du fait, gruger la clientèle des postes de traite situés en périphérie de cette région. Dès lors, à la clientèle de qui les commerçants mauriciens souhaitaient-ils s'attaquer? Évidemment, l'établissement des postes en Haute-Mauricie a eu tôt fait de nuire aux marchands établis à Trois-Rivières. Toutefois, des indices laissent croire que l'offensive visait plus particulièrement d'autres adversaires.

On pourrait croire que, rendu accessible aux commerçants à compter de 1774, le territoire de la Haute-Mauricie, comme l'ensemble de l'ancien territoire indien, constituait un espace attirant à partir duquel il devenait possible de concurrencer la Hudson's Bay Company. D'ailleurs, la compagnie anglaise était pleinement consciente du danger. Dès 1775, elle avait organisé des expéditions dans l'arrière-pays afin d'évaluer la faisabilité d'y ériger des avant-postes qui contreraient l'action des commerçants canadiens ${ }^{13}$. Les initiatives subséquentes, prises par la Hudson's Bay Company, montrent toutefois qu'elle était davantage préoccupée par les secteurs du lac Mistassini où un King's Post était depuis longtemps en opération, et du lac Waswanipi où la North West Company s'était installée en 1799. Elle se limita d'abord à établir un avant-poste au lac Neoskweskau en 1793 pour concurrencer celui de Mistassini, mais sans trop de succès ${ }^{14}$. Par la suite, au début des années 1810, elle construisit un autre poste au lac Mistassini pour faire opposition à celui de la North West Company, avec suffisamment de succès pour que les Canadiens quittent la région en $1817^{15}$. Bref, il sem-

13. Edwin E. Rich, The History of the Hudson's Bay Company, 1670-1870 (London, The Hudson's Bay Record Society, 1959), 2: 100-101; K. G. Davies, dir., Northern Quebec and Labrador Journals and Correspondence, 1819-1835 (London, The Hudson's Bay Record Society, 1963), 288-289, 292.

14. K. G. Davies, op. cit., 292-293.

15. Ibid., 295. 
ble que jusque dans les premières années du XIX $\mathrm{X}^{\mathrm{e}}$ siècle, la concurrence à laquelle faisait face la Hudson's Bay Company provenait principalement des commerçants canadiens établis dans le bassin hydrographique de la baie James. Sans exclure que ceux de la Haute-Mauricie aient pu drainer une partie des fourrures des chasseurs de la Terre de Rupert, leur menace ne semblait pas préoccuper outre mesure les hauts dirigeants de la compagnie anglaise.

Un procès-verbal de 1807, contenu dans les Minutes of the Transactions of the North West Company at Fort William, offre quelques indices sur la raison d'être des postes de la Haute-Mauricie. Il y est rapporté que: «A long conversation then followed relative to the St Maurice \& Kings Posts Departments, and the Wintering partners unanimously desired that the Outfit of the former should be in future so limited as to secure the Concern from loss, - that River being considered merely as a barrier to defend the borders of the neighbouring posts, and the Outfit thereto to be adapted to this purpose only ${ }^{16}$.» Ainsi, entre 1802 et 1807 , les établissements de la North West Company en Haute-Mauricie semblaient concurrencer les autres postes de la compagnie à l'intérieur du Domaine. Dès lors, on peut présumer que la possibilité de concurrencer les postes du Domaine explique en grande partie l'établissement des premiers postes de traite en Haute-Mauricie. À cet égard, l'examen de la localisation géographique de ceux-ci semble indiquer un lien étroit avec les postes du Domaine. Pour un, le poste de la Rivière-aux-Rats, au dire de Verreault, était situé à huit lieues au sud: «[... d'] une rivière nommé Ashtorogami (endroit où l'on fait des Canots), de six arpents de large, navigable pour des Canadiens; cette rivière monte quarante lieues dans le Nord et va joindre la rivière Métabishouan ${ }^{17} . \gg$ On sait qu'à l'embouchure de la rivière Métabetchouane se trouvait un important poste du Domaine qui, entre 1770 et 1805 , aurait toutefois été déplacé un peu plus au nord-ouest, à Pointe-Bleue ${ }^{18}$. Par ailleurs, le poste de Coocoocache, s'il a bien existé à cette époque, aurait été situé tout près de la rivière Windigo «qui vient du Nord, du côté du Lac St.-Jean ${ }^{19}$ ». En effet, il existait un important portage reliant la rivière Windigo, et peutêtre aussi la Trenche, avec la rivière au Saumon qui se déverse dans la rivière Ashuapmuchuane, d'où on pouvait rapidement rejoindre le

16. W. Stewart Wallace, dir., Documents Relating to the North West Company (Toronto, The Champlain Society, 1934), 248.

17. Témoignage de François Verreault, loc. cit.

18. Michelle Guitard, Des fourrures pour le roi au poste de Métabetchouan, Lac Saint-Jean (Québec, Ministère des Affaires culturelles, 1984), 110-111.

19. Témoignage de François Verreault, loc. cit. 
King's Post au lac du même nom ${ }^{20}$. Quant au poste de Kikendatch, la carte de Bouchette montre clairement que celui-ci, situé au lac du même nom, était à proximité de la rivière Kisikan qui, au terme d'un portage, menait au lac Shekoubish, voisin du lac Ashuapmuchuane. Même l'énigmatique Pisnoy Post aurait été situé à l'embouchure d'une route fluviale menant à la rivière Ashuapmuchuane. Seul le poste de Weymontachie semblait échapper à cette logique, du fait qu'il visait avant tout à attirer les autochtones chassant à la source des rivières du Lièvre et Ruban. D'ailleurs, il fut érigé en 1806 par la North West Company, plusieurs années après que celle-ci eut obtenu la location du Domaine.

En résumé, la majorité des postes de traite établis en Haute-Mauricie, à compter du dernier quart du XVIII ${ }^{\mathrm{e}}$ siècle, étaient situés à proximité de routes fluviales permettant aux autochtones de la région de se rendre facilement aux établissements du Domaine du roi. De même, on peut présumer que leur fonction consistait à la fois à retenir en HauteMauricie les autochtones de la région et, éventuellement, d'y attirer des habitués des King's Posts. Devenue locataire des King's Posts en 1802, la North West Company se faisait concurrence à elle-même en opérant à plein régime ses postes de la Haute-Mauricie. En ce sens, on comprend mieux sa décision de les recycler en simples surveillants responsables de protéger la chasse gardée que constituait le Domaine.

\section{LA COEXISTENCE DE DEUX MONOPOLES, 1821-1831}

Dans les années 1810, le commerce des fourrures était devenu une entreprise peu rentable pour la Hudson's Bay Company et la North West Company. La perturbation des marchés en raison des guerres napoléoniennes, de même que le coût élevé des opérations qu'entraînait la forte concurrence, finirent par rendre la fusion des deux compagnies inévitables ${ }^{21}$. En 1821, la Hudson's Bay Company «absorba» sa rivale, obtenant du coup un monopole sur le commerce des fourrures du Labrador au Pacifique. Les dirigeants de la compagnie anglaise purent alors instaurer une panoplie de mesures de rationalisation destinées à favoriser les profits. Ainsi, le nombre des postes et des employés fut grandement réduit, les prix demandés pour les articles de traite furent majorés

20. Gouvernement du Québec, Description of the Surveyed Townships and Explored Territories of the Province of Quebec (Québec, 1889), 473; E. Taché, Carte de la partie nord-ouest de la province de Québec, s'étendant de la vallée du lac St. Jean au lac Témiscamingue (Archives nationales du Canada, Division des cartes et plans, 1880), no 0019131.

21. G. D. Taylor et P. A. Baskerville, A Concise History of Business in Canada (Toronto, Oxford University Press, 1994), 90-93. 
alors que ceux offerts pour les fourrures furent réduits ${ }^{22}$, et les crédits accordés aux chasseurs autochtones, dans le sillon de la politique du «Ready-Barter System», furent beaucoup moins généreux ${ }^{23}$. Par ailleurs, pour assurer des revenus à long terme, la Hudson's Bay Company instaura des mesures de conservation des animaux à fourrure, et des castors en particulier ${ }^{24}$. Enfin, la distribution de présents aux autochtones fut restreinte ${ }^{25}$, alors que, dans la mesure du possible, la vente ou les dons d'alcool devaient cesser. Dans l'ensemble, ces mesures furent profitables puisque, sous la gouverne de George Simpson, la compagnie connut une prospérité sans précédent jusqu'en $1870^{26}$.

Le succès commercial de la Hudson's Bay Company s'explique aussi par une politique agressive de protection des frontières. En effet, malgré la disparition de son principal rival, la compagnie ne fut pas à l'abri des petits compétiteurs qui n'hésitaient pas à se rendre jusqu'au cœur de la Terre de Rupert pour commercer avec les autochtones. Aussi, dans le but de nuire à ces petits opposants et de protéger les zones intérieures riches en fourrures, la Hudson's Bay Company n'hésita pas à sacrifier les profits dans plusieurs régions frontalières: «The only means of repelling encroachments was a commercial policy that forced the intruders to absorb such losses on the frontiers that they lost the interest or ability to continue the contest, [...] prices were regulated by those of the opposition; the Company encouraged the Indians to hunt for every available fur; and liquor was dispensed whenever it was introduced by competitors ${ }^{27}$.» Galbraith a brossé un portrait général des contextes de concurrence qui ont prévalu après 1821 le long de la frontière de la Terre de Rupert, ainsi que des stratégies défensives déployées par la Hudson's Bay Company ${ }^{28}$. Les données tirées des

22. Frank Tough, «Indian Behavior, Exchange and Profits in Northern Manitoba During the Decline of Monopoly, 1870-1930», Journal of Historical Geography, 16 (1990): 389.

23. Charles A. Bishop, The Northern Ojibwa and the Fur Trade: an Historical and Ecological Study (Toronto, Holt, Rinehart and Winston of Canada, 1974), 118-119.

24. Arthur J. Ray, «Some Conservation Schemes of the Hudson's Bay Company, 1821-50: an Examination of the Problems of Resources Management in the Fur Trade», Journal of Historical Geography, 1,1 (1975): 49-68.

25. Daniel Francis et Toby Morantz, Partners in Furs. A History of the Fur Trade in Eastern James Bay, 1600-1870 (Kingston et Montréal, McGill-Queen's University Press, 1983), 123; Shepard Krech III, «The Trade of the Slavery and Dogrib at Fort Simpson in the Early Nineteenth Century», dans Shepard Krech III, dir., The Subarctic Fur Trade: Native Social and Economic Adaptations (Vancouver, University of British Columbia Press, 1984), 112.

26. Edwin E. Rich, op. cit., 2: 498.

27. John S. Galbraith, op. cit., 10-11; voir aussi Arthur J. Ray, The Canadian Fur Trade in the Industrial Age (Toronto, University of Toronto Press, 1990), 91.

28. John S. Galbraith, op. cit., 24-110. 
livres de comptabilité des postes de traite de Weymontachie en HauteMauricie permettent de documenter de façon plus détaillée non seulement la nature de l'opposition dans cette région, mais également la stratégie de la compagnie anglaise pour protéger son territoire le long de la frontière avec le Canada.

Ainsi, en 1821, la Hudson's Bay Company a pris possession des installations de la North West Company en Haute-Mauricie. Elle a choisi de maintenir en opération le poste de la Rivière-aux-Rats, qui fut désormais utilisé comme dépôt et relais à l'intention des postes situés en amont sur la rivière Saint-Maurice ${ }^{29}$. À Weymontachie, où la Hudson's Bay Company était installée depuis l'hiver 1820-1821 $1^{30}$, l'abandon d'un des deux postes semblait aller de soi. Or, il en fut tout autrement. Peu après la passation des pouvoirs, des employés de l'ancien poste de la North West Company reprirent possession de l'établissement et s'affilièrent aux locataires des King's Posts. Un scénario semblable s'est probablement déroulé à La Tuque où un poste de la King's Posts Company était aussi en opération au cours des années 1820, forçant la Hudson's Bay Company à s'y établir en $1828^{31}$. En pratique, le Domaine du roi venait d'englober une partie du bassin septentrional de la rivière SaintMaurice.

En dépit de son engagement à ne pas nuire au commerce des locataires des King's Posts, la Hudson's Bay Company s'est affairée, tout au long des années 1820, à encercler le Domaine avec des postes de traite destinés à drainer les fourrures hors de ce territoire et à accroître le rendement de ses propres établissements de l'est de la baie James et du Labrador $^{32}$. On pourrait croire que c'est dans ce cadre qu'elle érigea, à l'hiver de 1824-1825, un poste de traite au lac Obedjiwan ${ }^{33}$, d'autant plus qu'on apprend que, l'année suivante, elle était en compétition directe avec un King's Post au même endroit ${ }^{34}$. Or, contrairement à la situation qui prévalait au début du XIX ${ }^{\mathrm{e}}$ siècle, la Hudson's Bay Company était maintenant beaucoup plus sensible à la concurrence exercée

29. Hudson's Bay Company Archives (HBCA), B. 230/d/2, Weymontachingue Account Book, 1823-1825; Journal of the St. Maurice Exploring Party, loc. cit.

30. HBCA, B. 230/d/1, Weymontachingue Account Book, 1821-1823.

31. Joseph Bouchette père, The British Dominions in North America (London, Longman, 1932); Journal of the St. Maurice Exploring Party, loc. cit.

32. R. Harvey Fleming, dir., Minutes of Council, Northern Department of Rupert Land, 1821-31 (Toronto, The Champlain Society, 1940), xlii, xliv; Edwin E. Rich, op. cit., 2: 433.

33. HBCA, B.230/d/2, loc. cit., fos 25, 29; HBCA, B. 230/e/1, Weymontachingue Report on District 1831 , fo 4.

34. HBCA, B. 230/d/6, Weymontachingue Account Book 1825-1828, fo 6; HBCA, B. 134/ c/2, Montreal Correspondence Inward, fo 79. 
par les petits commerçants indépendants dans le Haut Saint-Maurice. Aussi, la fonction principale du poste d'Obedjiwan - et aussi du poste de Weymontachie dans une certaine mesure - était avant tout de protéger la frontière de la Terre de Rupert contre les incursions croissantes des Canadiens ${ }^{35}$. À cet effet, Cuthbert Cumming, le chef du district du Saint-Maurice pour la Hudson's Bay Company, mentionnait en 1831: «[...] this Post [Obedjiwan] I consider to be of considerable importance not as a trading station, for very little is done in that way but from its proximity to the $\mathrm{H}$ Bay Company's territory ${ }^{36}$.» De même, les employés du poste avaient comme consigne d'encourager les autochtones à chasser de façon intensive dans le secteur «as an exhausted frontier is the best protection we can have against the encroachments of rival traders from Canada ${ }^{37} \gg$.

Parallèlement, les King's Post jouaient en Haute-Mauricie un rôle somme toute identique à celui des postes de la Hudson's Bay Company et à celui qu'avaient joué les postes de la North West Company antérieurement. Comme le résumait une fois de plus Cumming: «The stake they [King's Posts] play for is of the greatest importance to them - nothing less than the protection \& security of there trade on the frontiers of the Kings Posts - which otherways would be exposed to our encroachement ${ }^{38}$.» Dans l'ensemble, durant une dizaine d'années, l'opposition entre les deux compagnies fut féroce et coûteuse, au point où, en 1831, George Simpson, le gouverneur de la Hudson's Bay Company, considéra plus avantageux de louer le Domaine que de lui faire concurrence $^{39}$. Pourtant, en Haute-Mauricie, la Hudson's Bay Company avait pratiquement réussi à neutraliser ses adversaires. En 1829, elle s'apprêtait à leur donner le coup de grâce au lac Obedjiwan ${ }^{40}$ et, à Weymontachie, le chef de district pouvait écrire: «We have completely succeeded in monopolizing the trade [...] their expulsion from the district is inevitable ${ }^{41}$.» Jusqu'en 1831, les employés des King's Posts de la Haute-Mauricie continuèrent à entraver les opérations de la Hudson's Bay Company par diverses tactiques parfois fructueuses ${ }^{42}$. Toutefois, sur le plan strictement commercial, cette dernière semble avoir nette-

\footnotetext{
35. HBCA, B. 230/e/1, loc. cit., fo 4 .

36. Ibid.

37. R. Harvey Fleming, op. cit., lvi; Edwin E. Rich, op. cit., 2: 432-433.

38. HBCA, B. 230/e/1, loc. cit., fo 2 .

39. R. Harvey Fleming, op. cit., liii.

40. HBCA, B. 230/b/1, Weymontachingue Correspondence Book 1829, fo 3 .

41. Ibid., fos 1-2; HBCA, B. 134/c/4, Montreal Correspondence Inward, fo 340.

42. HBCA, B. 134/c/8, Montreal Correspondence Inward, fo 228.
} 
ment eu le dessus. Lorsque le locataire des King's Posts se résigna finalement à céder le bail du Domaine à la Hudson's Bay Company pour la somme de $25000 £^{43}$, celle-ci allait dès lors être en mesure d'établir en Haute-Mauricie, comme elle l'avait fait ailleurs à compter de 1821, son monopole sur le commerce des fourrures.

\section{LES MODALITÉS DE LA CONCURRENCE}

$\mathrm{Si}$, dans les années 1820 , la présence des postes de traite en HauteMauricie semblait davantage justifiée par des considérations d'ordre stratégique, il n'en demeure pas moins que l'acquisition de fourrures demeurait une priorité et, sur ce plan, une forte concurrence a prévalu. Nuire au travail des opposants devenait une nécessité et diverses tactiques ont été déployées, à cet égard, par les employés des deux compagnies. C'est ainsi que tant à l'hiver qu'à l'été, ils avaient recours aux «dérouines», une pratique ancienne qui consistait à se rendre aux campements des chasseurs afin de mettre la main plus rapidement, et avant les rivaux, sur les fourrures ${ }^{44}$. Pour espérer contrer l'adversaire à ce jeu, il fallait épier scrupuleusement ses moindres gestes ${ }^{45}$. Aussi, il était fréquent que des employés partent en canot avant le lever du jour afin de ne pas être repérés par l'opposition ${ }^{46}$. Or, en 1827 , le commis du poste de la Hudson's Bay Company à Weymontachie se résignait à constater que «the OP have not yet missed any of the men that I send off as far as I can learn ${ }^{47} \gg$. La nécessité de garder le concurrent à l'œil amenait les commerçants à s'établir à proximité les uns des autres. C'est ainsi qu'au début des années 1820, la Hudson's Bay Company érigea son poste de Weymontachie à moins d'un kilomètre de celui de la North West Company ${ }^{48}$. De même, si on en croit la carte de Bouchette, les postes de la Hudson's Bay Company et de la King's Posts Company au lac Obedjiwan auraient été construits à courte distance l'un de l'autre.

Un autre procédé commun visait à convaincre les employés d'un opposant de changer de camp. En Haute-Mauricie, les exemples d'enga-

43. R. Harvey Fleming, op. cit., liv.

44. HBCA, B. 230/a/1, Weymontachingue Post Journal 1827, fos 6, 9; John Ingall, op. cit., 134; John Adams, «Sketches of the Tête de Boule Indians, River St. Maurice», Transactions of the Literary and Historical Society of Quebec, 2 (1831): 28-29.

45. HBCA, B.230/a/1, loc. cit., fo 8 .

46. Ibid., fo 9 .

47. Ibid.

48. John Ingall, op. cit., 130; Joseph-Étienne Guinard, Mémoires d'un Père oblat (Montréal, 1945), texte manuscrit conservé aux Archives provinciales des oblats de Marie-Immaculée, 74. 
gés qui ont déserté leur employeur pour joindre les rangs de l'opposition sont nombreux ${ }^{49}$. Ces déserteurs pouvaient être attirés par la promesse de meilleures conditions de travail, d'une meilleure situation ou simplement d'une rémunération plus généreuse ${ }^{50}$. Au dire de Cumming, le manque de loyauté de ses employés, souvent attirés par la libéralité et les meilleurs salaires offerts par l'opposition, était l'un des principaux obstacles à une gestion efficace ${ }^{51}$. Dans la seconde moitié des années 1820, la King's Posts Company payait ses employés en moyenne $37 £$ par année, comparativement à $24 £$ pour la Hudson's Bay Company ${ }^{52}$. Enfin, à l'extrême, le recours à la violence, sous diverses formes, fut parfois envisagé. Des cas d'intimidation ${ }^{53}$, de séquestration ${ }^{54}$ et peutêtre de meurtres ${ }^{55}$ ont été rapportés en Haute-Mauricie au plus fort de la lutte.

Malgré tout, entraver le travail des rivaux ne constituait qu'un premier pas. Encore et surtout fallait-il convaincre les autochtones de céder leurs fourrures. Car ceux-ci décidaient finalement à qui iraient les fruits de leur chasse. Ce qui leur importait avant tout était ce qu'ils pouvaient obtenir non seulement en échange des fourrures, mais aussi en guise de présents et d'avances de la part des commis. Aussi, la politique de prix, le système de crédit et la distribution gratuite d'alcool et de tabac constituaient autant d'outils dont disposaient les commis pour constituer et conserver une clientèle fidèle dans leur poste. Sur ce plan, la concurrence bénéficiait habituellement aux autochtones qui obtenaient à la fois plus pour leurs fourrures, une marge de crédit plus ample et des présents en plus grande quantité. Par contre, pour les commerçants, cela se traduisait souvent par de faibles profits, sinon par des pertes.

À première vue, des indices laissent croire que les commis des deux compagnies en Haute-Mauricie ont effectivement fait preuve de libéralité à l'égard des autochtones et que ceux-ci en auraient largement profité. L'exemple des présents est particulièrement éloquent. Il était fréquent de voir un chasseur qui, ayant reçu gratuitement une certaine

49. HBCA, B. 134/c/8, loc. cit., fo 196.

50. HBCA, B. 134/c/2, loc. cit., fo 333; HBCA, B. 134/c/8, loc. cit., fos 248-249.

51. HBCA, B. 230/e/1, loc. cit., fo 3 .

52. HBCA, B. 230/z/1, Weymontachingue Miscellaneous Items 1824-1850, fo 2; HBCA, B. 230/d/7, Weymontachingue Account Book 1828-1829, fos 76-77.

53. HBCA, B. 134/c/4, loc. cit., fos 223-224.

54. HBCA, B. 134/c/8, loc cit., fo 221; Copy of the Record of Conviction of Moïse Villeneuve, JCABC (1831-1832): app. A); Letter to the Right Hon. Lord Viscount Goderich, London, 22nd October 1831. Ibid.

55. HBCA, B. 134/c/2, loc. cit., fo 79; Joseph-Étienne Guinard, op. cit., 75. 
quantité d'alcool à son arrivée au poste de la Hudson's Bay Company, traversait presque aussitôt au King's Post pour bénéficier, là aussi, de la générosité du commis ${ }^{56}$. Par ailleurs, entre 1825 et 1827 , la grande majorité des chasseurs de Weymontachie avaient un compte à la fois au poste de la Hudson's Bay Company et à celui de la King's Posts Company ${ }^{57}$.

On pourrait croire que ces faits témoignent à la fois d'un rapport de force relativement égal entre les deux compagnies, de même que d'un opportunisme manifeste de la part des autochtones. La réalité était cependant plus nuancée. En effet, on constate que, bien qu'ils aient pratiquement tous eu un compte aux deux postes de traite, les chasseurs de Weymontachie n'ont effectué que 23,2\% de leurs transactions au King's Post entre 1825 et 1830 (tableau 1$)^{58}$. De plus, entre 1825 et 1827 , un chasseur se procurait en moyenne, chaque année, pour $18 £$ d'articles de traite au King's Post. Ce montant ne représentait qu'environ le tiers du total de ses «dépenses» annuelles qui se chiffraient en moyenne à $51 £$ (tableau 2). Bien sûr, il y eut des exceptions. Au total, de 1825 à 1830, Joachim a effectué $48,1 \%$ de ses achats au King's Post alors que, de juillet 1825 à juin 1826, Caspoyan et Neweashish y ont dépensé respectivement $75,6 \%$ et $64,4 \%$ plus qu'au poste de la Hudson's Bay Company. Toutefois, dans l'ensemble, ce dernier poste a nettement eu la faveur des chasseurs.

Il est possible que cet écart considérable et surprenant soit dû en partie à une image incomplète des activités commerciales qui se sont déroulées au poste de la King's Posts Company. En effet, les livres de comptabilité de cette compagnie n'ont pas été tenus avec toute la rigueur qui caractérisait l'administration du poste rival. Des transactions ont ainsi pu être omises, surtout celles qui se déroulaient en forêt lors des dérouines. Cependant, c'est surtout à partir de l'été de 1828 que les livres du King's Post paraissent moins garnis. Il a été souligné plus haut qu'à cette époque, le poste de la Hudson's Bay Company semblait avoir gagné la bagarre commerciale qui l'opposait au poste rival en se ralliant la presque totalité des chasseurs. Néanmoins, pour les années 1825 à

56. HBCA, B. 230/a/1, loc. cit., fos 5, 7, 9.

57. HBCA, B. 230/d/4, Weymontachingue Account Book 1824-1827; HBCA, B. 230/d/6, loc. cit.

58. L'ensemble des données statistiques qui apparaissent dans les pages qui suivent ont été compilées à partir des livres de comptabilité suivants: B. 230/d/4, B. 230/d/6, B. 230/d/8 et B. 230/ $\mathrm{d} / 14$. Ces données sont associées à un échantillon représentatif de dix chasseurs autochtones de Weymontachie dont l'ensemble des transactions effectuées aux deux postes de traite entre 18241830 ont été inventoriées et analysées. 
TABLEAU 1

RÉPARTITION MENSUELLE DES TRANSACTIONS EFFECTUÉES PAR DIX CHASSEURS DE WEYMONTACHIE AUX POSTES DE LA HUDSON'S BAY COMPANY (HBC) ET DE LA KING'S POSTS COMPANY (KPC) À WEYMONTACHIE, DU $1^{\text {er }}$ JUILLET 1825 AU 30 JUIN 1830

$\begin{array}{lrrr} & \text { HBC } & \text { KPC } & \text { Total } \\ \text { Janvier } & 49 & 5 & 54 \\ \text { Février } & 24 & 17 & 41 \\ \text { Mars } & 31 & 6 & 37 \\ \text { Avril } & 8 & 4 & 12 \\ \text { Mai } & 31 & 12 & 43 \\ \text { Juin } & 128 & 36 & 164 \\ \text { Juillet } & 18 & 5 & 23 \\ \text { Août } & 54 & 14 & 68 \\ \text { Septembre } & 76 & 13 & 89 \\ \text { Octobre } & 87 & 35 & 122 \\ \text { Novembre } & 29 & 14 & 43 \\ \text { Décembre } & 25 & 10 & 35 \\ \text { Total } & 560 & 171 & 731\end{array}$

Source: Hudson's Bay Company Archives (HBCA), B. 230/d/4, 6, 8, 14.

1827, pour lesquelles les données du King's Post semblent plus fiables, on constate qu'un chasseur se rendait sept fois sur dix au poste de la Hudson's Bay Company et qu'il y achetait pour une valeur $29 \%$ supérieure à ce qu'il dépensait au poste rival (tableau 2).

Comment expliquer cette nette préférence des autochtones envers la Hudson's Bay Company ${ }^{59}$ ? Cette compagnie offrait-elle des produits d'une meilleure qualité ${ }^{60}$ ? Offrait-elle plus de tabac ou d'alcool en guise

59. On ne peut certes pas invoquer ici un attachement traditionnel à cette compagnie en raison d'une éventuelle présence plus ancienne dans la région. En fait, les deux compagnies s'étaient implantées en Haute-Mauricie à la même époque. De plus, si une compagnie avait dû bénéficier d'un avantage sur ce plan, c'est la King's Post Company qui exploitait à Weymontachie un poste établi depuis le tournant du XIXe siècle.

60. Les données explicites manquent en ce qui concerne la qualité des articles de traite de chacune des compagnies en Haute-Mauricie. Toutefois, à la même époque à Mistassini, il arrivait 
de présents ${ }^{61}$ ? Dans ce dernier cas, on constate que, de 1825 à 1827 , au plus fort de la lutte entre les deux compagnies, un chasseur se rendait en moyenne 2,9 fois par an au King's Post contre 7,6 fois au poste de la Hudson's Bay Company. De plus, à chaque visite, il achetait en moyenne pour $6 £$ au premier poste, contre $4 £$ au second. Aussi, comme chaque visite était une occasion d'obtenir gratuitement du tabac ou de l'alcool, le fait qu'on se rendait plus souvent au poste de la Hudson's Bay Company, en achetant chaque fois pour un petit montant, pourrait indiquer que les présents y étaient distribués plus généreusement qu'à l'autre poste. Or, même si tel était le cas, un tel phénomène ne peut fournir qu'une partie de la réponse. En fait, des indices plus révélateurs semblent se dégager lorsqu'on analyse la mise en application des politiques de prix et des systèmes de crédit dans les deux postes de Weymontachie.

\section{LES POLITIQUES DE PRIX}

Dans les années 1820, les hautes instances de la Hudson's Bay Company avaient pour habitude de fixer le prix des produits offerts dans leurs postes de traite, de même que celui des fourrures qu'y échangeaient les autochtones, en tenant compte, dans chaque cas, de leur valeur sur le marché européen. On connaît mal cependant ce sur quoi les locataires du Domaine se basaient pour définir leur politique de prix. Or, dans la réalité, les politiques de prix dictées par les dirigeants étaient souvent inapplicables sur le terrain, car chaque poste de traite évoluait dans un univers particulier où les commis devaient composer avec une foule de contraintes. Parmi les plus fréquentes, il y avait le fait

fréquemment que des clients du poste de la Hudson's Bay Company se rendent au King's Post d'Ashuapmuchuan afin d'obtenir, le plus souvent, des pièges en métal. Or, le fait que ces chasseurs se procuraient rarement d'autres articles à ce King's Post pourrait indiquer que la marchandise que leur offrait la Hudson's Bay Company, à l'exception de quelques articles, était à tout le moins de qualité égale, et peut-être supérieure à celle des King's Post.

61. La distribution de tabac et d'alcool avait été l'un des principaux outils de concurrence entre la Hudson's Bay Company et la North West Company. Si, à compter de 1821, la première compagnie a fortement diminué les quantités d'alcool et de tabac offertes aux chasseurs dans les régions où elle jouissait d'un véritable monopole, dans les secteurs de forte concurrence, comme en Haute-Mauricie, elle n'a généralement eu d'autre choix que de maintenir la distribution de tels produits. Par ailleurs, une des raisons pour lesquelles la Hudson's Bay Company distribuait gratuitement de l'alcool et du tabac était que ces articles n'étaient pas essentiels pour la subsistance des autochtones, la compagnie préférant garder les outils de prédations, les articles utilitaires et les vêtements et les tissus comme monnaie d'échange contre les fourrures. Ce n'était donc pas des articles qui liaient un chasseur au poste de traite, de sorte que pour gagner la faveur des chasseurs, il fallait se montrer toujours plus généreux que le concurrent. Voir Toby Morantz, «"So Evil a Practice": a Look at the Dept System in the James Bay Fur Trade», dans Rosemary M. Ommer, dir., Merchant Credit and Labour Strategies in Historical Perspective (Fredericton, Acadiensis Press, 1990), 209. 
TABLEAU 2

\section{NOMBRE DE VISITES ET MONTANT DES ACHATS ANNUELS AUX POSTES DE LA HUDSON'S BAY COMPANY ET DE LA KING'S POSTS COMPANY À WEYMONTACHIE, DU $1^{\text {er }}$ JUILLET 1825 AU 30 JUIN 1827}

Chasseurs

$\begin{array}{lccrc} & \text { Visites } & \begin{array}{c}\text { Achats } \\ \text { (en pences) }\end{array} & \text { Visites } & \begin{array}{c}\text { Achats } \\ \text { (en pences) }\end{array} \\ \text { Nabawish } & 16 & 26562 & 11 & 18222 \\ \text { Menisino } & 23 & 39235 & 7 & 9168 \\ \text { Caspoyan } & 10 & 8187 & 11 & 21181 \\ \text { Quataweq } & 13 & 11310 & - & - \\ \text { Samabane } & 11 & 12112 & 2 & 2430 \\ \text { Joachim } & 17 & 11555 & 9 & 13711 \\ \text { Oskelaway } & 12 & 16919 & 1 & 2778 \\ \text { Majeshk, fils } & 22 & 16752 & 7 & 5755 \\ \text { Naweashish } & 11 & 6716 & 9 & 8166 \\ \text { Majeshk, père } & 16 & 9933 & 1 & 6360 \\ \text { Moyenne annuelle } & 7,6 & 7964(33 £) & 2,9 & 4389(18 £)\end{array}$

Hudson's Bay Company

1825-1827
King's Posts Company

$1825-1827$

par chasseur

Source: HBCA, B. 230/d/4, 6.

que les autochtones n'appréciaient généralement pas que le coût des produits de traite augmente ${ }^{62}$. Par ailleurs, en situation de concurrence, il fallait offrir plus aux autochtones pour leurs fourrures. Enfin, les fourrures et les articles de traite n'étaient pas tous de même qualité et leur prix devait souvent être négocié. Aussi, un commis devait recourir à diverses ruses pour parvenir à réaliser des profits ${ }^{63}$.

62. Arthur J. Ray, Indians in the Fur Trade: Their Role as Trappers, Hunters, and Middlemen in the Lands Southwest of Hudson Bay (Toronto et Buffalo, University of Toronto Press, 1974), 62.

63. Ibid., 62-63; Arthur J. Ray et Donald Freeman, «Give Us Good Measure»: an Economic Analysis of Relations Between the Indians and the Hudson's Bay Company Before 1763 (Toronto, University of Toronto Press, 1978), 95, 170. 
Sur le plan des contraintes au profit, le contexte commercial qui prévalait en Haute-Mauricie dans les années 1820 comportait une autre variable. Tant les postes de la Hudson's Bay Company que ceux de la King's Posts Company avaient avant tout pour fonction d'assurer la protection respective de la Terre de Rupert et du Domaine du roi. Pour les compagnies, des profits dans la région auraient sans doute été bienvenus, mais elles s'attendaient plutôt à des pertes et elles étaient prêtes à les assumer. À cet égard, ce portrait du fonctionnement du King's Post de Weymontachie, brossé par Cumming, est éloquent:

[...] if we take into consideration the very extravagant manner in which the trade [of the K. P. C.] in this River is conducted, the great number of men uselessly employed the exorbitant wages allowed them - the triffling amount of their returns we [?] with perfect safty presume that they sustain a considerable yearly loss but on the other hand it may be observed that the revenus arising from the monopoly of that fine Country the Kings Posts - enables them to support any loss incurred here ${ }^{64}$.

Par conséquent, le rôle des commis en Haute-Mauricie n'était pas tant d'assurer la rentabilité de leur établissement que de gérer efficacement la mobilité des chasseurs le long des frontières. Cette gestion devait passer, autant que possible, par l'attachement des autochtones à un poste particulier, afin qu'ils ne tombent pas sous l'influence de l'opposition, ce qui ouvrirait la porte à des incursions en territoires protégés. Pour parvenir à cette fin, un des principaux appâts utilisés était le prix demandé pour les articles de traite, de même que celui offert pour les fourrures des chasseurs.

Les livres de comptabilité des postes de Weymontachie montrent que, sur le plan du prix des articles de traite, les commis fonctionnaient avec des prix standard qui étaient sensiblement les mêmes aux deux établissements. Toutefois, en y regardant de plus près, on remarque que les commis bénéficiaient tout de même d'une marge de manœuvre dans l'application de ces prix. En réalité, non seulement le prix d'un article pouvait varier d'un chasseur à l'autre, mais il pouvait également varier pour un même chasseur à l'intérieur d'une même année. Par exemple, en 1829 , alors que Quataweq payait sa livre de tabac 180 pences au poste de la Hudson's Bay Company, le vieux Majesk payait la sienne 60 pences au même endroit. En février 1825, Nabawish a payé 80 pences pour sa livre de plomb alors qu'en juin de la même année, au même poste, une quantité identique lui coûta 120 pences. De tels exemples sont fré-

64. HBCA, B. 230/e/1, loc. cit., fo 2 . 
TABLEAU 3

ÉCARTS ENTRE LES PRIX STANDARDS ET LES PRIX MOYENS DEMANDÉS* DE QUELQUES ARTICLES DE TRAITE DANS LES POSTES DE WEYMONTACHIE, 1825-1829

\begin{tabular}{|c|c|c|c|c|c|}
\hline Articles & Compagnies & Types de prix & $1825-1826^{* *}$ & $1826-1827$ & $1827-1828$ \\
\hline \multirow[t]{4}{*}{ Poudre (livre) } & $\mathrm{HBC}$ & Prix standard & 60 & 60 & 60 \\
\hline & & Prix demandés & 60 & 53 & 60 \\
\hline & KPC & Prix standard & 60 & 60 & 60 \\
\hline & & Prix demandés & 84 & 90 & 60 \\
\hline \multirow[t]{4}{*}{ Plomb (livre) } & $\mathrm{HBC}$ & Prix standard & 20 & 20 & 20 \\
\hline & & Prix demandés & 13 & 16 & 13 \\
\hline & KPC & Prix standard & 30 & 30 & 30 \\
\hline & & Prix demandés & 76 & 36 & 30 \\
\hline \multirow[t]{4}{*}{ Tabac (livre) } & $\mathrm{HBC}$ & Prix standard & 60 & 60 & - \\
\hline & & Prix demandés & 60 & 39 & - \\
\hline & KPC & Prix standard & 60 & 60 & 60 \\
\hline & & Prix demandés & 60 & 60 & 45 \\
\hline
\end{tabular}

quents dans les livres de comptes de la Hudson's Bay Company et ils montrent que lorsque le commis s'éloignait des prix standard, c'était habituellement à la baisse (tableau 3). Par contre, le commis du King's Post est resté beaucoup plus fidèle aux prix standard et, lorsqu'il s'en écartait, c'était le plus souvent à la hausse.

Par ailleurs, à l'image du prix demandé pour les articles de traite, celui offert pour les fourrures a également varié. Une fois de plus, les commis des deux postes de Weymontachie fonctionnaient à partir de prix standard qui étaient sensiblement les mêmes aux deux postes et qui n'ont pas varié de 1825 à 1830 . Toutefois, comme dans le cas des articles de traite, les commis ont joui d'une marge de manœuvre dans l'application des prix des fourrures. En comparant les prix standard de celles-ci avec les prix moyens offerts aux chasseurs, on constate qu'en de rares occasions les seconds ont dépassé les premiers (tableau 4). Habituellement, on offrait le prix standard ou moins. De plus, en com- 


\section{TABLEAU 4}

\section{ÉCARTS ENTRE LES PRIX STANDARDS ET LES PRIX MOYENS OFFERTS* POUR LES FOURRURES DANS LES POSTES DE TRAITE DE WEYMONTACHIE, 1825-1829}

\begin{tabular}{|c|c|c|c|c|c|c|}
\hline Fourrures & Compagnies & Types de prix & $1825-26$ & $1826-27$ & $1827-28$ & $1828-29$ \\
\hline Castors & $\mathrm{HBC}$ & Prix standard (hiver) & 180 & 180 & 180 & 180 \\
\hline \multirow[t]{3}{*}{ (d'hiver) } & & Prix offerts (hiver) & 166 & 149 & 150 & 178 \\
\hline & $\mathrm{KPC}$ & Prix standard (hiver) & 180 & 180 & 180 & 180 \\
\hline & & Prix offerts (hiver) & 140 & 165 & 180 & 180 \\
\hline Loutres & $\mathrm{HBC}$ & Prix standard (hiver) & 180 & 180 & 180 & 180 \\
\hline \multirow[t]{3}{*}{ (d'hiver) } & & Prix offerts (hiver) & 195 & 210 & 180 & 210 \\
\hline & $\mathrm{KPC}$ & Prix standard (hiver) & 180 & 180 & 180 & 180 \\
\hline & & Prix offerts (hiver) & 180 & 180 & 180 & 180 \\
\hline $\begin{array}{l}\text { Rats } \\
\text { musqués }\end{array}$ & $\mathrm{HBC}$ & Prix standard (hiver) & 15 & 15 & 15 & 15 \\
\hline \multirow[t]{3}{*}{ (d'hiver) } & & Prix offerts (hiver) & 15 & 14 & 15 & 15 \\
\hline & $\mathrm{KPC}$ & Prix standard (hiver) & 15 & 15 & 15 & 15 \\
\hline & & Prix offerts (hiver) & 15 & 15 & 15 & 15 \\
\hline \multirow[t]{4}{*}{ Martres } & $\mathrm{HBC}$ & Prix standard & 30 & 30 & 30 & 30 \\
\hline & & Prix offerts & 20 & 26 & 30 & 30 \\
\hline & $\mathrm{KPC}$ & Prix standard & 30 & 30 & 30 & 30 \\
\hline & & Prix offerts & 24 & 30 & 30 & 30 \\
\hline
\end{tabular}

parant le prix moyen de chaque type de fourrure offert par les deux commis, il appert que le King's Post offrait un prix plus élevé ou égal à celui de la Hudson's Bay Company. Ainsi, au cours des deux années intenses de concurrence, à savoir de 1825 à 1827, le King's Post a offert en moyenne $8 \%$ de plus que le poste rival pour les fourrures des chasseurs. Toutefois, en prenant en considération que le commis du King's Post demandait, à l'inverse, $23,8 \%$ de plus que le commis de la Hudson's Bay Company pour ses articles de traite, un chasseur bénéficiait, finalement, d'un pouvoir d'achat près de $16 \%$ plus élevé à ce dernier poste de traite. 


\section{LE CRÉDIT}

Le crédit fut une des composantes les plus importantes du commerce des fourrures. La raison d'être des postes de traite ayant été d'obtenir des fourrures, il était dans l'intérêt des marchands d'aider les autochtones dans leurs activités prédatrices. Cette «assistance» se présentait habituellement sous la forme d'avances accordées aux chasseurs chaque automne. On leur donnait, à cette époque de l'année, des articles nécessaires non seulement pour le piégeage des animaux à fourrures, mais aussi pour la chasse de subsistance et la vie quotidienne. Toutefois, cette distribution d'articles ne se faisait qu'à la condition que les chasseurs reviennent au poste au printemps suivant pour rembourser leur dette avec les fourrures obtenues durant l'hiver. Le crédit constituait donc un outil qui assurait aux commerçants des entrées continues de fourrures dans leurs postes de traite. Bref: «[...] credit provided an economic safety net for native and trader alike since both of them depended on regular returns. In addition, company traders used the dept to establish a claim on some or all of an Indian's future returns. This was a major concern whenever local competition was keen ${ }^{65}$.» Tout au long des années 1820, la Hudson's Bay Company et la King's Posts Company ont eu recours au système de crédit en Haute-Mauricie. Compte tenu du rôle stratégique des postes de traite dans cette région, le crédit constituait pour les commis non seulement une nécessité pour l'acquisition des fourrures, mais également un outil privilégié pour gérer la mobilité des autochtones. Théoriquement, une fois qu'un chasseur avait contracté un crédit dans un poste, il y demeurait attaché. Les livres de comptabilité de la Hudson's Bay Company montrent qu'entre 1824 et 1830 , son poste de Weymontachie a conservé une clientèle fidèle et régulière. Sans prétendre que le crédit explique tout, on peut affirmer qu'il a sans aucun doute constitué un facteur déterminant dans cet attachement au poste. Ainsi, entre le 5 juillet 1824 et le 22 juin 1829 , ce n'est qu'à trois occasions seulement que des chasseurs de notre échantillon ont remboursé au complet leur crédit à la compagnie lors d'une transaction $^{66}$. Le reste du temps, tous sont demeurés endettés envers la compagnie. Le somme de leur crédit pouvait cependant varier, de quelques livres à plusieurs dizaines ${ }^{67}$. En moyenne, un chasseur maintenait un crédit annuel de $30 £$ (tableau 5).

65. Arthur J. Ray, «The Decline of Paternalism in the Hudson's Bay Company Fur Trade, 1870-1945», dans Rosemary E. Ommer, dir., op. cit., 189.

66. HBCA, B. 230/d/4, loc. cit., fos 11, 37, 48.

67. Ibid., fo 117 . 
TABLEAU 5

\section{ENDETTEMENT MOYEN ANNUEL * PAR CHASSEUR AU POSTE DE LA HUDSON'S} BAY COMPANY À WEYMONTACHIE, 1825-1829

$\begin{array}{lrrrrc}\text { Chasseurs } & 1825-26 & 1826-27 & 1827-28 & 1828-29 & \text { Moyenne annuelle } \\ \text { Nabawish } & 6105 & 7860 & 13320 & 18555 & 11460 \\ \text { Menisino } & 10369 & 8341 & 9689 & 17161 & 11390 \\ \text { Caspoyane } & 8372 & 7954 & 8247 & 13032 & 9401 \\ \text { Quataweq } & 3856 & 4268 & 4378 & 7750 & 5053 \\ \text { Samabane } & 4504 & 4900 & 8665 & 7721 & 6448 \\ \text { Joachim } & 7757 & 9126 & 8926 & 10266 & 9019 \\ \text { Oskelaway } & 4641 & 3343 & 3837 & 11043 & 5716 \\ \text { Majeshk, fils } & 3390 & 5223 & 5519 & 5312 & 4861 \\ \text { Naweasit } & 6278 & 3936 & 6328 & 8634 & 6294 \\ \text { Majeshk, père } & 2439 & 2111 & 3478 & 1912 & 2485 \\ \text { Moyenne par } & 5771 & 5706 & 7239 & 10139 & 7214(30 £) \\ \text { chasseur } & & & & & \\ \text { *Valeur exprimée en pences. } & & & & \\ \text { Source: HBCA, B. 230/d/4, } 8 . & & & & \end{array}$

Pour ce qui était du King's Post, les données sont beaucoup moins fiables. C'est uniquement pour les années 1825-1826 et 1826-1827 que celles-ci sont suffisamment nombreuses, sans pour autant être absolument fiables, pour offrir un aperçu de l'importance du crédit (tableau 6). On constate qu'un chasseur y maintenait en moyenne, chaque année, un crédit de $17 £$. En comparant avec le crédit annuel moyen durant les mêmes années au poste de la Hudson's Bay Company qui se chiffrait à $23 £$, on constate que ce dernier établissement consentait un crédit $35 \%$ plus élevé. Cet avantage, combiné à une politique de prix plus avantageuse, explique probablement en grande partie le fait que les chasseurs autochtones de Weymontachie aient nettement préféré commercer au poste de la Hudson's Bay Company.

Par ailleurs, les chasseurs autochtones ont su tirer profit du système de crédit sur un autre plan. En effet, il semble que les cycles de crédit en vigueur dans les postes de Weymontachie n'aient pas fonctionné de manière parallèle, mais plutôt en contre-pointe. La répartition mensuelle des transactions d'achats des chasseurs montre que celles effec- 
TABLEAU 6

ENDETTEMENT MOYEN ANNUEL ${ }^{*}$ PAR CHASSEUR AU POSTE DE LA KING'S POSTS COMPANY À WEYMONTACHIE, 1825-1828

$\begin{array}{lrrr}\text { Chasseurs } & 1825-26 & 1826-27 & \text { Moyenne annuelle } \\ \text { Nabawish } & 2645 & 7929 & 5287 \\ \text { Menisino } & 5556 & 6600 & 6078 \\ \text { Caspoyane } & 4925 & 12958 & 8942 \\ \text { Quataweq } & - & - & - \\ \text { Samabane } & 912 & (201) & 356 \\ \text { Joachim } & 3021 & 9080 & 6051 \\ \text { Oskelaway } & 5293 & 4816 & 5055 \\ \text { Majeshk, fils } & 3327 & 3720 & 3524 \\ \text { Naweasit } & 2010 & 5210 & 3610 \\ \text { Majeshk, père } & 4597 & 2565 & 3581 \\ \text { Moyenne par chasseur } & 3229 & 5268 & 4248(17 £) \\ \text { *Valeur exprimée en pences. } & & & \\ \text { Source: HBCA, B. 230/d/6, 14. } & & & \end{array}$

tuées au poste de la Hudson's Bay Company étaient plus nombreuses en juin qu'à tout autre mois de l'année (tableau 1). Toutefois, c'était en septembre et en octobre que les achats les plus importants, en termes de coût, étaient effectués (tableau 7). Par contre, la situation qui prévalait au King's Post était différente. Si les chasseurs fréquentaient également ce poste de manière plus intensive en juin, c'était aussi durant ce mois qu'ils effectuaient le plus gros de leurs achats. Parallèlement, on constate que, pour chacun des postes de traite, le moment de l'année où les crédits contractés par les chasseurs étaient les plus élevés coïncidait avec celui des plus gros achats: en septembre et octobre au poste de la Hudson's Bay Company, et en juin au King's Post.

En ce qui concerne les habitudes de remboursement, c'était en juin que les chasseurs se présentaient au poste de la Hudson's Bay Company avec le plus de fourrures et une autre pointe, moins prononcée, se présentait en septembre et octobre (tableau 7). En juin, les revenus tirés des fourrures étaient supérieurs au coût des achats. Aussi, compte tenu, à quelques exceptions près, que les autochtones avaient toujours un 
TABLEAU 7

PROFIL MENSUEL DE L'ENDETTEMENT* D'UN CHASSEUR AUX POSTES DE WEYMONTACHIE,

1825-1830

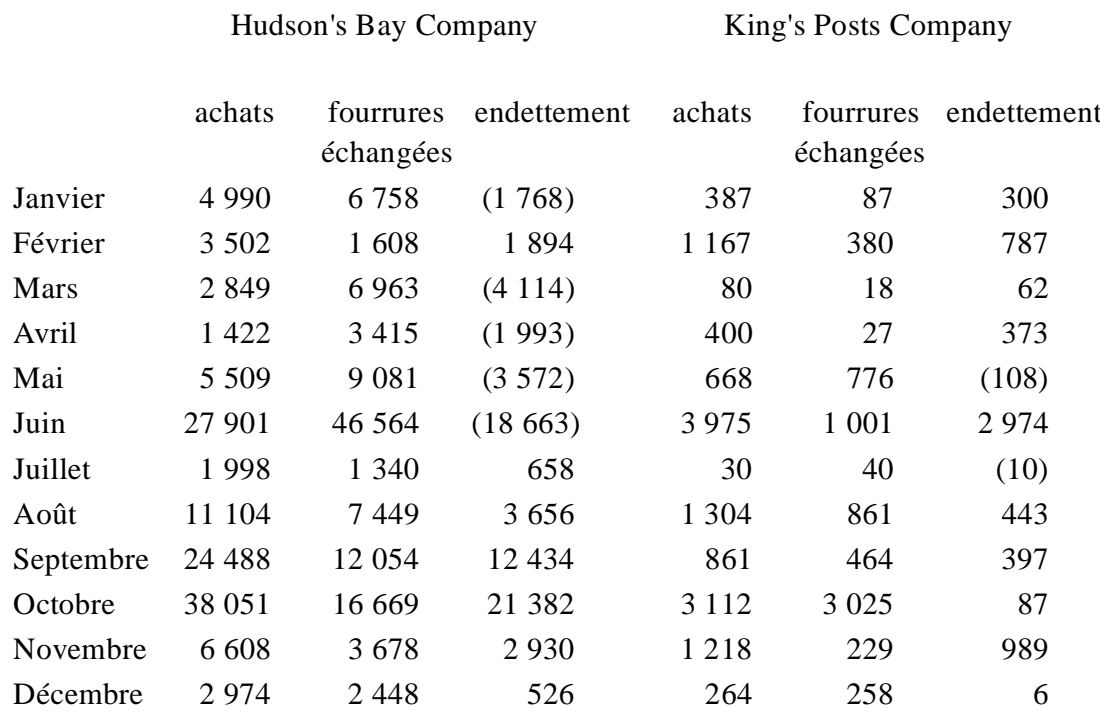

*Valeur exprimée en pences.

Source: HBCA, B. 230/d/4, 6, 8, 14.

compte déficitaire, on peut présumer qu'ils remboursaient, en juin, le crédit contracté l'automne précédent et qu'ils faisaient également des achats pour la saison estivale, lesquels étaient supérieurs en valeur à ce qui restait comme fourrures après l'acquittement du crédit automnal. En septembre et en octobre, les dépenses étaient cependant supérieures aux revenus. Les fourrures échangées à cette époque auraient donc servi à rembourser le crédit estival et payer une partie des achats automnaux. Un scénario différent prévalait au King's Post. Dans ce cas, c'était en octobre que les chasseurs y échangeaient le plus de fourrures, avec une autre pointe, plus faible, en juin. C'est dire qu'en juin, les chasseurs remboursaient environ les deux tiers de leur crédit automnal et effectuaient des achats importants pour l'été. À l'automne, une chasse estivale apparemment fructueuse permettait de rembourser la majeure 
partie du crédit estival et, parfois, une partie du crédit automnal. Autrement dit, les chasseurs de Weymontachie semblaient considérer le King's Post comme un lieu d'approvisionnement privilégié pour l'été, alors que le poste de la Hudson's Bay Company était plutôt apprécié pour les achats d'hiver. Ainsi, les deux cycles de crédit semblent avoir été complémentaires. De retour de son terrain de chasse en juin, un chasseur rapportait l'essentiel de ses fourrures au poste de la Hudson's Bay Company où il remboursait son crédit hivernal et achetait une certaine quantité d'articles à crédit. Il traversait ensuite au King's Post où il achetait à crédit une panoplie d'articles de traite qui complétaient les achats effectués à l'autre poste. Au début de l'automne, il échangeait une bonne part des fourrures obtenues de sa chasse d'été au King's Post pour acquitter son crédit contracté en juin, puis traversait au poste rival pour contracter son gros crédit hivernal. Comme la Hudson's Bay Company octroyait un crédit plus ample et des prix moins élevés pour ces articles, il était donc avantageux de s'y procurer un crédit hivernal car la saison de chasse exigeait l'achat d'articles en grande quantité. Par contre, une fois ce crédit acquitté à la Hudson's Bay Company en juin, il restait peu à offrir pour se procurer le nécessaire pour passer l'été. Ainsi, en contractant un crédit au King's Post, un chasseur pouvait accroître la quantité de ses achats. Évidemment, il aurait été possible à un chasseur de ne pas contracter de crédit estival au King's Post, d'acheter à crédit au poste de la Hudson's Bay Company et d'y transiger, à l'automne, le fruit de sa chasse d'été. C'est apparemment ce que faisait le chef Quataweq. Par contre, en fréquentant les deux postes, on s'assurait une plus grande marge de crédit globale et on profitait des présents offerts aux deux endroits.

\section{CONCLUSION}

Dans les années 1820 , le rôle stratégique des postes de traite de la Hudson's Bay Company et de la King's Posts Company en Haute-Mauricie a eu pour conséquence de placer les rapports économiques entre commerçants et autochtones dans un climat de libéralité qui, de toute évidence, a bénéficié à chaque parti. De leur côté, les compagnies ont apparemment réussi à garder en Haute-Mauricie les chasseurs de la région, protégeant leurs chasses gardées respectives, à savoir la Terre de Rupert et le Domaine du roi. Toutefois, pour y arriver, il aura fallu tricher sur le plan des politiques de prix officielles et se montrer généreux sur le montant des crédits. Mais, de toute évidence, il s'agissait-là de manœuvres calculées. Je n'ai relevé aucun indice montrant que les com- 
mis exercèrent des pressions sur les autochtones afin qu'ils remboursent leur crédit. De plus, des produits au coût élevé tels que les fusils, la farine, le maïs et l'alcool étaient abondamment cédés à crédit aux autochtones chaque automne. La vente de ces produits à la fin du printemps ou à l'été, lorsque les autochtones possédaient un fort volume de fourrures, aurait constitué une bonne façon de limiter l'endettement des chasseurs de même que des pertes éventuelles ${ }^{68}$.

Toutefois, sur le plan strictement économique, la concurrence s'est avérée très coûteuse pour les commerçants. Outre les pertes engendrées par des articles vendus à bas prix, l'opération des postes de traite coûtait cher. Par exemple, chaque compagnie devait compter sur un nombre élevé d'employés, payés à fort prix ${ }^{69}$, pour aller relancer les autochtones en pleine forêt. En 1825, la King's Posts Company employait près d'une trentaine d'hommes en Haute-Mauricie ${ }^{70}$ et la Hudson's Bay Company en comptait plus d'une quarantaine à la fin des années $1820^{71}$. À titre de comparaison, cette dernière n'engagea rarement plus d'une dizaine d'employés pour son district du Saint-Maurice après les années 1820, alors qu'elle jouissait d'un monopole dans la région.

S'il est possible qu'entre 1775 et 1802, les marchands indépendants puis la North West Company ait pu tirer des revenus intéressants de la traite en Haute-Mauricie, à compter de cette dernière date, des considérations stratégiques de même que l'arrivée de la Hudson's Bay Company ont sans doute limité le rendement des postes mauriciens. On ne peut chiffrer avec exactitude les revenus ou les pertes de la King's Posts Company. Toutefois, si on en croit Cumming, cité plus haut, non seulement elle aurait connu des pertes, mais celles-ci étaient probablement importantes. La situation n'a pas été plus enviable pour la Hudson's Bay Company. Notamment, certaines tactiques pour contrer l'opposition ont eu leur prix. Par exemple, les tarifs élevés offerts pour les fourrures à Obedjiwan ont eu pour conséquence non seulement de gruger la clientèle des autres postes de la compagnie situés dans les bassins hydrographiques de la baie James et de l'Outaouais supérieur ${ }^{72}$, mais aussi de mettre cette clientèle en contact avec les exploitants du King's Post.

68. Jacques Frenette, Mingan au 19e siècle: cycles annuels des Montagnais et politiques commerciales de la Compagnie de la Baie d'Hudson (Ottawa, Musées nationaux du Canada, Service canadien d'ethnologie, dossier no 106, 1986), 53.

69. HBCA, B. 230/e/1, loc. cit., fo 2 .

70. HBCA, B. $230 / \mathrm{d} / 6, l o c$. cit.

71. HBCA, B. 230/d/7, loc. cit., fos 76-77; Gilbert P. de T. Glazebrook, The Hargrave Correspondence, 1821-1843 (Toronto, The Champlain Society, 1938), 47.

72. John S. Galbraith, op. cit., 30. 
D'ailleurs, le poste d'Obedjiwan était considéré comme peu rentable, notamment en raison de l'incompétence de ses dirigeants ${ }^{73}$, ce qui profitait à l'opposition. Aussi, bien qu'en 1829 le district du Saint-Maurice ait été qualifié de prospère ${ }^{74}$, le responsable de la Hudson's Bay Company à Weymontachie écrivait l'année suivante: «I continued to attend to the trifling business of the place [...] we are strongly opposed here by the Lessees of the King's Posts a persevering and vigilant set - progress under such circumstances cannot be expected at least need not be expected ${ }^{75} . »$

73. HBCA, B. 230/e/1, loc. cit., fo 4

74. HBCA, B.230/b/1, loc. cit., fo 3 .

75. Gilbert P. de T. Glazebrook, op. cit., 47. 